\title{
CRITERIA FOR SELF-ADJOINTNESS OF THE DIRAC OPERATOR ON PSEUDOMANIFOLDS
}

\author{
ARTHUR W. CHOU
}

(Communicated by Jonathan M. Rosenberg)

\begin{abstract}
We study the Dirac operator on pseudomanifolds with piecewise constant curvature metric. The criteria for the self-adjointness of the Dirac operator are obtained and a vanishing theorem is proved. At the end we make some comments on the index theorem and the $\widehat{A}$-genus.
\end{abstract}

\section{INTRODUCTION}

Recently there has been a great deal of interest in the Dirac operator on singular spaces (see, for example, [4], [13], [15], and [22]). In this paper we extend some of our results in [14] for spaces with conical singularities to pseudomanifolds. In a way the analysis there is "hard" and the analysis here "soft". For the background materials on the Dirac operator and spinors we refer to [1], [14], [17], [18], [20] and [21]. For pseudomanifolds the general references are [10], [11] and [16]. The corresponding theory of the Laplacian and the signature operator on singular spaces and pseudomanifolds is developed by Cheeger in [9-12]. Our approach is based on his.

We now briefly recall some notions in [14], [10] and [11]. Let $N$ be a closed riemannian manifold of dimension $m$ with metric $g$. By the metric cone on $N, C(N)$, we mean the space $(0, \infty) \times N$ equipped with the metric $d r \otimes d r+r^{2} g$ where $r \in(0, \infty)$. Set

$$
C_{0, u}(N)=\{(r, x) \in C(N) ; 0<r<u\} .
$$

Definition. $X$ is called a space with isolated metrically conical singularities if there exist points $p_{j}$ in $X, j=1,2, \ldots, k$ such that $X \backslash \bigcup\left\{p_{j}\right\}$ is a smooth riemannian manifold and each $p_{j}$ has a neighborhood $U_{j}$ such that $U_{j} \backslash\left\{p_{j}\right\}$ is isometric to $C_{0, u}(N)$ for some $u$ and cross section $N$.

Received by the editors October 24, 1988 and, in revised form, December 5, 1988.

1980 Mathematics Subject Classification (1985 Revision). Primary 58G11, 58G12, 58G25; Secondary $53 \mathrm{C} 20$.

Key words and phrases. Dirac operators, singular spaces, pseudomanifolds.

This research is supported in part by the National Science Foundation under Grant No. MCS8301627. 
Without loss of generality we assume that $X=C_{0,1}(N) \cup M$ where $N=\partial M$ and the union is along the boundary.

Notice that $X \backslash \bigcup\left\{p_{j}\right\}$ is an incomplete (smooth) manifold and the situation is completely different from that of a compact or complete manifold. Let $D_{0}$ denote the Dirac operator on the space of smooth spinors with compact support on $X$ and $D$ denote the Dirac operator with domain $\{\phi ; \phi$ is smooth and in $L^{2}$ and $D \phi$ is also in $\left.L^{2}\right\}$, and $\bar{D}$ denote its $L^{2}$ closure. Let $\widetilde{D}$ denote the Dirac operator on the cross section $\{1\} \times N=N$. In [14] we used separation of variables techniques and a priori estimate of the behavior of spinors near the singularity to prove

Theorem 1.1. The Dirac operator $\bar{D}$ is self-adjoint on the space of square-integrable spinors on $X$ if and only if there is no eigenvalue of $\widetilde{D}$ whose absolute value is strictly less than $1 / 2$.

Let “*" denote the $L^{2}$ adjoint of an operator. Consider two self-adjoint Dirac Laplacians,

$$
\Delta_{D}=\bar{D}_{0}^{*} \cdot \bar{D}_{0} \text { and } \Delta_{N}=\bar{D}^{*} \cdot \bar{D}
$$

corresponding to Dirichlet and Neumann boundary conditions, respectively. Since $\bar{D}_{0}^{*}=\bar{D}$, it follows that if the eigenvalues $\mu$ of $\widetilde{D}$ satisfy $|\mu| \geq 1 / 2$, then $\bar{D}=\bar{D}_{0}$ and $\Delta_{D}=\Delta_{N}$. Even then the Dirac Laplacian can fail to be essentially self-adjoint due to the "limit circle" phenomena (see [11] [14]).

Definition. $\phi$ is called a harmonic spinor if $\phi$ is in $L^{2}$ and $\dot{D} \phi=D^{2} \phi=0$.

Note that on a singular space it is possible that $D^{2} \phi=0$ but $D \phi \neq 0$ (see [14]).

It is a consequence of the Lichnerowicz-Bochner-Weitzenböck formula ([19]) that if the dimension of $N$ is greater than or equal to two and the scalar curvature of $N$ is greater than or equal to that of a sphere, then the eigenvalues $\mu$ of $\widetilde{D}$ satisfy $|\mu| \geq 1 / 2$. In [14] we also proved the following general fact.

Proposition 1.2. Let $Y$ be any (possibly incomplete) spin manifold. Suppose that the scalar curvature $\kappa$ of $Y$ has a lower bound; i.e., $\kappa$ does not go to $-\infty$. Then we have

$$
\|D \theta\|^{2} \geq\|\nabla \theta\|^{2}+\frac{1}{4} \cdot \int_{Y} \kappa\|\theta\|_{y}^{2}
$$

for all $\theta$ in the domain of $\bar{D}_{0}$, where $\nabla$ is the connection, \|\| denotes the global $L^{2}$-norm, and \|\|$_{y}$ the pointwise norm at a point $y$.

Combining these facts we arrived at the following vanishing theorem:

Theorem 1.3. Let $X$ be a space with isolated metrically conical singularities. If the dimension of $X$ is greater than or equal to three, and the scalar curvature $\kappa$ of $X$ satisfies $\kappa \geq 0$, then $\bar{D}$ is self-adjoint and all harmonic spinors $\theta$ are parallel ; i.e., $\nabla \theta=0$. 
Moreover if, in addition to $\kappa \geq 0, \kappa>0$ somewhere on $X$ then $\operatorname{Kernel}\left(\bar{D}^{*}\right.$. $\bar{D})=\operatorname{Kernel}(\bar{D})=\{0\}$; i.e., there are no harmonic spinors on $X$.

In fact these results in [14] are invariant under quasi-isometry.

Definition. A metric $g$ is quasi-isometric to a metric $g^{\prime}$ if there exists constants $c$ and $d$ such that $c g \leq g^{\prime} \leq d g$.

Therefore we can consider a more general class of singularities.

Definition. $X$ is called a space with isolated conical singularities if there exist points $p_{j}$ in $X, j=1,2, \ldots, k$ such that $X \backslash \bigcup\left\{p_{j}\right\}$ is a smooth riemannian manifold and each $p_{j}$ has a neighborhood $U_{j}$ such that $U_{j} \backslash\left\{p_{j}\right\}$ is quasiisometric to $C_{0, u}(N)$ for some $u$ and cross section $N$.

Our proofs in [14] show that the above theorems can be generalized to this class of metrics, which is essential for our later discussions of pseudomanifolds.

Corollary 1.4. The above Theorem 1.1 and Theorem 1.3 hold for spaces with isolated conical singularities.

Although isolated conical singularities are rather special, they are general enough for our purposes in dealing with pseudomanifolds. Recall that an $n$ dimensional pseudomanifold $X^{n}$ is a finite simplicial complex such that every point is contained in a closed $n$-simplex and every $(n-1)$-simplex is the face of at most two $n$-simplices. This is a general class of singular spaces. For instance it contains all (triangulated) algebraic varieties. For simplicity, we assume that $X$ is a closed oriented normal pseudomanifold; that is, a compact oriented pseudomanifold without boundary such that the link of each simplex in the codimension two skeleton is connected; i.e., a circle (see [16]). Our results also hold for pseudomanifolds which are not normal, see the remark at the end of $\S 2$. Let $\Sigma^{i}$ denote the $i$-dimensional skeleton of $X$. Then $X^{n} \backslash \Sigma^{n-2}$ : the nonsingular part of $X$, is a smooth (incomplete) manifold homotopically equivalent to the dual 1-skeleton. Since its second cohomology group vanishes, $X^{n} \backslash \Sigma^{n-2}$ is always a spin manifold. This fact is particularly interesting because it enables us to apply the analytic techniques of the Dirac operator on spinors to study the geometry and topology of pseudomanifolds or piecewise-linear manifolds which are not necessarily spin.

We assume that $X^{n} \backslash \Sigma^{n-2}$ is equipped with a metric such that for each $n$ simplex the curvature in the interior is a (fixed) nonnegative constant $K$ and the faces are totally geodesic. We will emphasize the case that $X^{n} \backslash \Sigma^{n-2}$ has a piecewise flat metric $g$; i.e., $K=0$. This is because the other metrics $g^{\prime}$ with constant curvature $K(K \geq 0)$ are quasi-isometric to $g$, and our results remain true under quasi-isometries. But the case $K>0$ does occur since the links of simplices inherit metrics of this type.

Here by the link, $L\left(\sigma^{k}\right)$, of a simplex $\sigma^{k}$ in $X$, we mean the following. Let $p$ be an interior point of $\sigma^{k}$. Then $L\left(\sigma^{k}\right)$ is the boundary of a sufficiently 
small metric ball of radius $\varepsilon$ about $p$, in the direction normal to $\sigma^{k}$, with metric rescaled (multiplied by $1 / \varepsilon$ ) so that it is isometric to the boundary of the same ball but with radius 1 . Assume that $X$ is piecewise flat. Then $L\left(\sigma^{k}\right)$ is an $(n-k-1)$-dimensional pseudomanifold made up of spherical simplices of constant curvature 1 with totally geodesic faces. Now consider the union of line segments (or geodesics), emanating from $p$ and normal to $\sigma^{k}$, of a sufficiently small length $\varepsilon$. This set is isometric to a finite cone $C_{0, \varepsilon}\left(L\left(\sigma^{k}\right)\right)$ on the link $L\left(\sigma^{k}\right)$. We call $C\left(L\left(\sigma^{k}\right)\right)$ the normal cone to $\sigma^{k}$. Thus the point $p$ has a neighborhood isometric to $U^{k} \times C_{0, \varepsilon}\left(L\left(\sigma^{k}\right)\right)$. This is what makes our previous analysis on conical singularities relevant. And since the analysis of the Dirac operator is completely "local" we can use inductive argument to generalize the results on conical singularities to pseudomanifolds.

To carry out the inductive argument successfully, one needs to examine the metrics near the singularities inside the links $L\left(\sigma^{k}\right)$. It is easy to see that they are asymptotically metrically conical. That is, the metrics are of the form

$$
d r \otimes d r+h(r)^{2} g \quad \text { where } h(r)=r+O\left(r^{2}\right) \text { as } r \text { goes to } 0 ;
$$

and hence they are quasi-isometric to the metrically conical singularity. A detailed study of the elliptic operators related to such metrics can be found in [6-8]. But in this paper, Corollary 1.4 suffices.

In $\S 2$ we outline the inductive argument and use it to obtain the criteria for self-adjointness (Theorem 2.2) and some vanishing theorems (Theorem 2.3, Corollary 2.5) for the Dirac operator. In $\S 3$ we discuss the issues related to the index theorems and $\widehat{A}$-genus.

\section{SELF-ADJOINTNESS OF THE DIRAC OPERATOR AND VANISHING THEOREMS}

We first investigate the cone on the one dimensional link, the circle $S^{1}$. This case is not considered in Theorem 1.3 and turns out to be crucial. $S^{1}$ has two spin structures: one is the trivial 2-fold covering, the other the nontrivial one. Surprisingly the nontrivial spin structure on $S^{1}$ is the one induced from the disk, and hence a spin boundary. On the other hand, the trivial spin structure does not bound any spin manifold (see [18] and [20]). Therefore the bundle of spinors on $S^{1}$ induced from the disk is (topologically) the nontrivial line bundle on $S^{1}$. It follows that

Proposition 2.1. Suppose that $X$ is a closed two dimensional space with conical singularity, $X=C_{0,1}\left(S^{1}\right) \cup M$. Then the Dirac operator is self-adjoint if and only if

(1) the spin structure on the cross section $S^{1}$ is nontrivial; and

(2) the length of the cross section $S^{1}$, normalized to be at distance 1 from the cone tip, is less than or equal to $2 \pi$.

Proof. By Theorem 1.1, we need to find conditions which guarantee that the eigenvalues of the Dirac operator $\widetilde{D}$ on the circle are greater than or equal to 
$1 / 2$. If the spin structure is trivial, then the constant function 1 is in the kernel of $\widetilde{D}$, and hence there is an eigenvalue 0 . Therefore the spin structure must be nontrivial. The Dirac operator acts like $i d / d \theta$ locally. Since the bundle of spinors is twisted, the smallest eigenvalue is $\pi /$ length $\left(S^{1}\right)$ and therefore (2) follows. Q.E.D.

This leads to the following definition.

Definition. Let $X$ be any pseudomanifold. A spin structure on $X^{n} \backslash \Sigma^{n-2}$ is called regular if the induced spin structures on all the 1-dimensional links are nontrivial. Otherwise it is called irregular.

Any smooth (triangulated) spin manifold with codimension two skeleton removed carries a regular spin structure, the induced one from the smooth manifold; and generally it also carries irregular spin structures. Any pseudomanifold admits a regular spin structure by extending the regular spin structure on 1dimensional links through homotopy. Now we are ready to prove

Theorem 2.2. Let $X$ be a closed oriented normal pseudomanifold with a metric of piecewise constant curvature $K, K \geq 0$. Suppose that the spin structure on the nonsingular part of $X$ is regular. Then the Dirac operator $D$ is self-adjoint if and only if the length of each link of the codimension two skeleton is less than or equal to $2 \pi$.

Proof. We will outline the proof and refer to [11] and [14] for the technical details. As in the proof of Theorem 1.1 ([14]), it suffices to check that

$$
\langle D \theta, \phi\rangle=\langle\theta, D \phi\rangle \quad \text { for any } \theta \text { and } \phi \text { in the domain of } D \text {. }
$$

The proof is by induction on the dimension of $X$. Central to the inductive argument are the following two observations.

(1) The first is a combinatorial one. As before, let $L\left(\sigma^{k}, p\right)$ denote the (normalized) link of $\sigma^{k}$ around a point $p$. It is a pseudomanifold with a metric quasi-isometric to the flat one. In the inductive argument, we need to consider the links of the simplices in $L\left(\sigma^{k}, p\right)$ and the links in those links, $\ldots$, etc., etc.. But we claim that all these links are quasi-isometric to links of some simplices "in $X$ ". In order to see this, consider a three dimensional piece " $A B C D$ " in Figure 1 in a three dimensional pseudomanifold. The link of " $p$ " is a 2-dimensional " abcd", suitably normalized (see $\S 1$ ). Now the link of the point " $a$ " in that link " abcd" is the (normalized) 1-dimensional circle " $b c d$ " which is isometric to the link of the 1-simplex " $A p$ " in " $A B C D$ ". Such iterated links are pseudomanifolds of dimension smaller than $X$ and carry a metric with curvatures $\geq 1$, which is quasi-isometric to the flat metric.

(2) The second observation is that the Fredholm theory holds for the Dirac operator $D$ on pseudomanifolds with piecewise constant curvature metric (that is, the Green's operator is compact, and hence the eigenvalues are discrete and the eigenfunctions are $L^{2}$-complete). This follows from the fact that the heat 


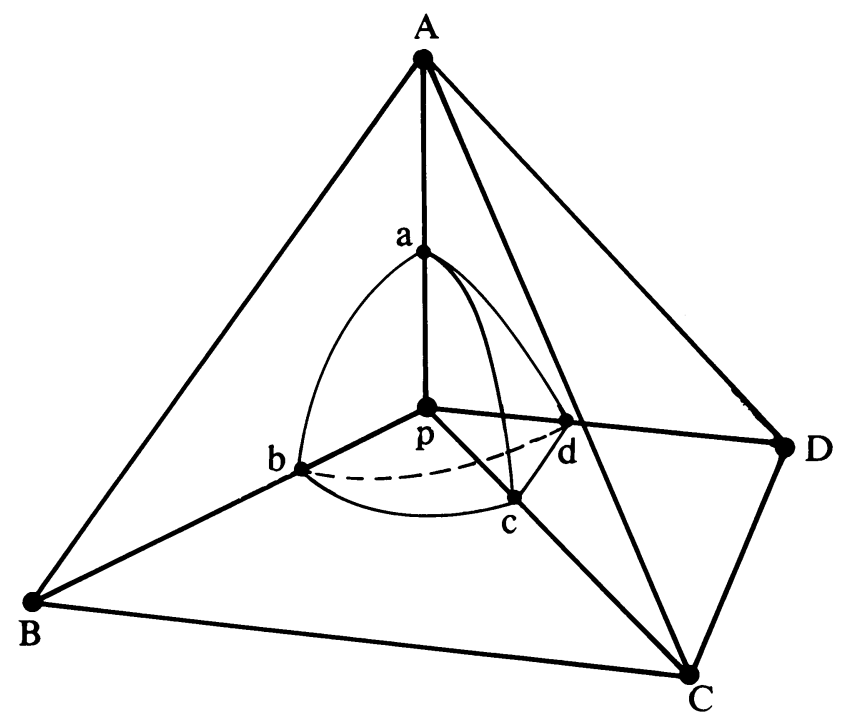

kernel is of trace class, which can be proved inductively as in [11] and [14]. In fact, Fredholm theory for each dimension of $X$ can be verified inductively and jointly within the present proof, right after the self-adjointness of $D$ is first established in that dimension. (Otherwise we need to choose "ideal boundary conditions". That is what we want to avoid here.) This double induction works because the proof of the self-adjointness will use the Fredholm theory only for the cross section of the cone, which has smaller dimension. In each dimension, the proof of the heat kernel being of trace class follows exactly the same line as in [11] and [14] and will not be repeated here. This observation allows us to apply the same separation of variables technique as the one used in the proof of Theorem 1.1 to prove a generalized version of it; that is, replacing the smooth cross section $N$ by pseudomanifolds. This (generalized) theorem also holds if we consider metrics quasi-isometric to the conical one instead (see Corollary 1.4).

We now carry out the inductive steps.

The base case that $\operatorname{dim} X=2$ is true by Proposition 2.1. Assume that the theorem is true for $X$ of dimension less than or equal to $n$. Now suppose that $\operatorname{dim} X=n+1$. As explained in the introduction, $X$ can be decomposed into $U^{k} \times C_{0, \varepsilon}\left(L\left(\sigma^{k}\right)\right)$ whose union is $X$. Using the partition of unity with suitable cut-off functions, we can localize the analysis to each $U^{k} \times C_{0, \varepsilon}\left(L\left(\sigma^{k}\right)\right)$. Since there is a natural splitting of spinors on a product structure and $U^{k}$ is smooth, we need only to check (2.1) for spinors on the finite cone $C_{0, \varepsilon}\left(L\left(\sigma^{k}\right)\right)$, multiplied by a cut-off function which is identically 1 in a neighborhood of the cone tip and vanishes away from it. Some care is needed to choose the cut-off function, see [11, p. 618]. 
The cross section $L\left(\sigma^{k}\right)$ is a pseudomanifold of positive constant curvature $K \geq 1$. By the fact (1) above, it satisfies the conditions of the theorem and hence, by the inductive hypothesis, the Dirac operator $\widetilde{D}$ on the link $L\left(\sigma^{k}\right)$ is self-adjoint. Proposition 1.2 and the fact $\widetilde{D}=\widetilde{D}_{0}$ imply that the eigenvalues of $\widetilde{D}$ are all $\geq 1 / 2$. Therefore, by use of the generalized version of Theorem 1.1 as stated in the above observation (2), we conclude that for the Dirac operator $D$ on $C_{0, \varepsilon}\left(L\left(\sigma^{k}\right)\right)$,

$$
\langle D \theta, \phi\rangle=\langle\theta, D \phi\rangle
$$

where $\theta$ and $\phi$ are spinors on $C_{0, \varepsilon}\left(L\left(\sigma^{k}\right)\right)$ described above in the domain of $D$. By gluing the pieces $U^{k} \times C_{0, \varepsilon}\left(L\left(\sigma^{k}\right)\right)$ together, we prove the case $\operatorname{dim} X=n+1$. Q.E.D.

The vanishing theorems are easy consequences of this theorem.

Theorem 2.3. Let $X$ be a closed oriented normal pseudomanifold with a metric of piecewise constant curvature $K \geq 0$. If the spin structure is regular and each link of the codimension 2 skeleton has length less than or equal to $2 \pi$, then all harmonic spinors are parallel. If $K>0$ instead, then there is no harmonic spinor on $X$.

Proof. By Proposition 1.2, $K \geq 0$ implies that for harmonic spinors $\theta, \nabla \theta=$ 0 . If $K>0$, then $\kappa>0$ and $\theta$ must be identically zero for $\theta$ in the domain of $D$. Q.E.D.

Now let us recall an observation due to Gromov.

Lemma 2.4. Suppose that $X$ is a pseudomanifold with piecewise flat metric; i.e., $K=0$, and the length of each link of the codimension 2 skeleton is strictly less than $2 \pi$. Then there exists a metric with piecewise constant positive curvature $K^{\prime}\left(K^{\prime}>0\right)$ such that the length of each such link remains strictly less than $2 \pi$.

Proof. We can replace each flat simplex in $X$ with a simplex of constant curvature $K^{\prime}$ with the same edge length. If $K^{\prime}$ is sufficiently small, then the length of each such link can remain strictly less than $2 \pi$. Q.E.D.

Corollary 2.5. Let $X$ be a normal pseudomanifold with piecewise flat metric; i.e., $K=0$. If the spin structure is regular and the length of each link of the codimension 2 skeleton is strictly less than $2 \pi$, then there is no harmonic spinor on $X$.

Proof. Since the dimension of the kernel of the Dirac operator $D$ is invariant under quasi-isometry, this corollary follows immediately from the lemma. Q.E.D.

Remark. If $X$ is not normal, then a link of a codimension 2 cell can be a disjoint union of circles. If each of them has length $\leq 2 \pi$ (or $<2 \pi$ ), then the above Theorem 2.3 and Corollary 2.5 still hold. 


\section{COMMENTS ON THE INDEX THEOREMS}

We first recall the index theorems for isolated metrically conical singularities obtained in [14]. Let $D^{+}$and $D^{-}$denote the Dirac operator restricted to the space of (+)-spinors and (-)-spinors, respectively, on an even dimensional manifold (see [3] and [14]). In [14] we also discussed the self-adjoint extensions of $D_{0}$ by imposing the "ideal boundary condition" on the domain of the Dirac operator. One such extension is called $D_{b}$. We derived the following index formula. Let $\hat{A}(p)$ denote the $\hat{A}$-polynomial of Pontrjagin forms $\left\{p_{i}\right\}$ and $\eta(0)$ the eta-invariant of the Dirac operator on $N, \widetilde{D}$ (see [2]).

Theorem 3.1. Let $X^{2 k}=C_{0,1}(N) \cup M$ be a space with isolated metrically conical singularity. Then

$$
\begin{aligned}
& \operatorname{Index}\left(\bar{D}_{0}^{+}\right)=\int_{M} \hat{A}(p)+\frac{1}{2}(\eta(0)-h)-\sum_{0<\mu_{j}<1 / 2} \operatorname{dim}\left(E_{\mu_{j}}\right), \\
& \operatorname{Index}\left(\bar{D}^{+}\right)=\int_{M} \hat{A}(p)+\frac{1}{2}(\eta(0)+h)-\sum_{-1 / 2<\mu_{j}<0} \operatorname{dim}\left(E_{\mu_{j}}\right) \text { and } \\
& \operatorname{Index}\left(\bar{D}_{b}^{+}\right)=\int_{M} \hat{A}(p)+\frac{1}{2} \eta(0)
\end{aligned}
$$

where $h=\operatorname{dim} \operatorname{Ker}(\widetilde{D})$, and $E_{\mu_{j}}$ is the eigenspace of $\widetilde{D}$ with eigenvalue $\mu_{j}$.

It follows from the vanishing theorems (Theorem 2.3 and Corollary 2.5) that

Theorem 3.2. Let $X^{2 k}$ be a closed oriented pseudomanifold with a regular spin structure and a metric of piecewise constant curvature $K \geq 0$. Suppose that either (1) $K>0$ and each circle in the links of the codimension 2 skeleton has length $\leq 2 \pi$, or (2) $K=0$ and each of such circles has length $<2 \pi$. Then

$$
\operatorname{Index}\left(\bar{D}_{0}^{+}\right)=\operatorname{Index}\left(\bar{D}^{+}\right)=0 .
$$

On a pseudomanifold $X^{n}$ one can impose ideal boundary conditions on the conical singularities near the codimension 2 skeleton, $C\left(L\left(\sigma^{n-2}\right)\right)$, to acquire self-adjoint extensions. For example, let the domain of $D_{b}$ consist of $(+)$ spinors (i.e., those of the form $f(r)\left(\bar{\phi}+\frac{\partial}{\partial r} \cdot \bar{\phi}\right)$, see [14]) which behave like $O\left(r^{-1 / 2}\right)$ on $C\left(L\left(\sigma^{n-2}\right)\right)$ (i.e., $\left.f(r)=O\left(r^{-1 / 2}\right)\right)$ near the cone tip inside $X$, and (-)-spinors (i.e., those of the form $g(r)\left(\bar{\phi}-\frac{\partial}{\partial r} \cdot \bar{\phi}\right)$ ) which behave like $o\left(r^{-1 / 2}\right)$ (i.e., $g(r)=o\left(r^{-1 / 2}\right)$ ). Then it can be checked by integration by parts that $\bar{D}_{b}$ is self-adjoint on $X$. Using the heat equation method on pseudomanifolds in [11], we can derive the following index formula.

Theorem 3.3. On an even dimensional pseudomanifold with piecewise flat metric,

$$
\operatorname{Index}\left(\bar{D}_{b}^{+}\right)=\frac{1}{2} \cdot \sum_{\sigma^{0}} \eta\left(L\left(\sigma^{0}\right)\right),
$$


where $\eta\left(L\left(\sigma^{0}\right)\right)$ is the eta-invariant of the Dirac operator on the link of the 0dimensional cell $\sigma^{0}$.

Notice that if the length of each link of the codimension 2 skeleton is $\leq 2 \pi$ then $\bar{D}=\bar{D}_{0}=\bar{D}_{b}$ and formula (3.1) still holds.

The situation for $\bar{D}_{0}^{+}$and $\bar{D}^{+}$on general pseudomanifolds is rather unclear at this point, due to the contribution from the small eigenvalues of the Dirac operator on the links of $\Sigma^{n-2}$. Although the formula (3.1) is very similar to the one defining the $L$-class in [11], the ideal boundary condition is rather artificial and apparently devoid of any topological meaning. Anyhow, there is no canonical "index formula" for the Dirac operator on pseudomanifolds. This indicates that extending the $\widehat{A}$-genus to pseudomanifolds is difficult (see the open problem 3 on [5, p. 223]), and that both geometric and analytic tools are needed in order to clarify these issues.

\section{ACKNOWLEDGMENTS}

The author wishes to express his gratitude to Jeff Cheeger for inspiration and encouragement. $\mathrm{He}$ is grateful to Blaine Lawson for several very helpful discussions. Part of this work was done in 1983-84 during which time the author was a visiting member at the Institute for Advanced Study. He would like to thank the Institute for its hospitality.

\section{REFERENCES}

1. M. F. Atiyah, R. Bott and A. A. Shapiro, Clifford modules, Topology 3 (supp. 1) (1964), 3-38.

2. M. F. Atiyah, V. K. Patodi, and I. M. Singer, Spectral asymmetry and Riemannian geometry. I, Math. Proc. Camb. Philos. Soc. 77 (1975), 43-69.

3. M. F. Atiyah and I. M. Singer, The index of elliptic operator. III, Ann. of Math. (2) 87 (1968), 546-604.

4. J.-M. Bismut and J. Cheeger, Family index for manifolds with boundary; superconnections and cones (preprint), April 1988.

5. A. Borel et al., Intersection cohomology, Birkhäuser, Boston, 1984.

6. J. Brüning and R. Seeley, Regular singular asymptotics, Adv. Math. 58 (1985), 133-148.

7. _ The resolvent expansion for second order regular singular operators, J. Funct. Anal. $\mathbf{7 3}$ (1987), 369-429.

8. __, An index theorem for first order regular singular operators, Amer. J. Math. 110 (1988), 659-714.

9. J. Cheeger, On the spectral geometry of spaces with cone-like singularities, Proc. Nat. Acad. Sci. U.S.A. 76 (1979), 2103-2106. (A revised version is in preprint (1980).)

10. _ On the Hodge theory of riemannian pseudomanifolds, Proc. Sympos. Pure Math., vol. 36, Amer. Math. Soc. Providence, R. I., 1980, 91-145.

11. __ Spectral geometry of singular spaces, J. Differential Geometry 18 (1983), 575-657.

12. _ A vanishing theorem for piecewise constant curvature space, in Curvature and Topology of Riemannian Manifolds, Lecture Notes in Math. vol. 1201, Springer-Verlag Berlin-Heidelberg 1986, pp. 33-40.

13. _ , $\eta$-invariant, the adiabatic approximation and conical singularities, J. Differential Geometry 26 (1987), 175-221. 
14. A. Chou, The Dirac operator on spaces with conical singularities and positive scalar curvatures, Trans. Amer. Math. Soc. 289 (1985), 1-40.

15. P. Gerbert and R. Jackiw, Classical and quantum scattering on a spinning cone (preprint), July 1988.

16. M. Goresky an R. MacPherson, Intersection homology theory, Topology 19 (1980), 135-162.

17. N. Hitchin, Harmonic spinors, Advances in Math. 14 (1974), 1-55.

18. H. B. Lawson and M. L. Michelson, The geometry of spinors (manuscript).

19. A. Lichnerowicz, Spineurs harmoniques, C. R. Acad. Sci. Paris Ser. A-B 257 (1963), 7-9.

20. J. Milnor, Spin structure on manifolds, L'Enseignement Math. 9 (1963), 198-203.

21. _ Remarks concerning spin manifolds, Differential and Combinatorial Topology, Princeton Univ. Press, N. J., 1964, pp. 55-62.

22. E. Witten, Global gravitational anomalies, Comm. Math. Phys. 100 (1985), 197-229.

Department of Mathematics and Computer Science, Clark University, Worcester, MASSACHUSETTS 01610 